\title{
Drop when the stakes are high: adaptive, flexible use of dropping behaviour by aphids
}

\author{
Rosalind K. Humphreys ${ }^{\mathrm{a}, *}$, Graeme D. Ruxton ${ }^{\mathrm{a}}$ and Alison J. Karley ${ }^{\mathrm{b}}$ \\ ${ }^{a}$ School of Biology, University of St Andrews, Dyer's Brae House, St Andrews, \\ Fife KY16 9TH, UK \\ ${ }^{\mathrm{b}}$ The James Hutton Institute, Invergowrie, Dundee DD2 5DA, UK \\ *Corresponding author's e-mail address: rosalindkh08@gmail.com
}

Received 11 January 2021; initial decision 11 February 2021; revised 2 March 2021; accepted 10 March 2021; published online 30 March 2021

\begin{abstract}
For herbivorous insects, dropping from the host plant is a commonly-observed antipredator defence. The use of dropping compared to other behaviours and its timing in relation to contact with a predator was explored in both pea aphids (Acyrthosiphon pisum) and potato aphids (Macrosiphum euphorbiae). Pea aphids dropped more frequently in response to ladybird adults (Adalia bipunctata) than lacewing larvae (Chrysoperla carnea). Potato aphids mainly walked away or backed-up in response to both predator types; but they dropped more frequently relative to other non-walking defences when faced with ladybird adults. Contact with a predator was an important influencer of dropping for both species, and most drops occurred from adjacent to the predator. Dropping appears to be a defence adaptively deployed only when the risk of imminent predation is high; factors that increase dropping likelihood include presence of faster-foraging predators such as adult ladybirds, predator proximity, and contact between aphid and predator.
\end{abstract}

Keywords

Aphididae, defence, dropping behaviour, predator-prey interaction, risk assessment.

\section{Introduction}

The threat of predation is a major selective force for prey species, driving the evolution of morphological, physiological and behavioural antipredator adaptations that increase the likelihood of survival (Edmunds, 1974; Caro, 2005; Ruxton et al., 2018). However, the immediate risk of predation any prey individual faces will vary greatly over the course of its lifetime, chang- 
ing on seasonal, daily and even shorter timescales (Lima \& Dill, 1990). The effectiveness of any behavioural antipredator defence at enhancing a prey individual's fitness can be expected to vary depending on the context of specific predator-prey interactions, and so these adaptations should be flexibly deployed. Behavioural decision-making should reflect the trade-offs between the relative costs and benefits of any behaviour compared to the alternatives available to a prey individual, the evaluation of which will depend on a wide range of ecological variables.

Dropping is an antipredator behaviour that is common and taxonomically widespread across the animal kingdom. It involves the passive or active release from a substrate (or loss of powered motion) such that an animal's escape from an imminent threat is powered by gravity, wind or water currents (Humphreys \& Ruxton, 2019). The obvious benefit of such a defence is immediate and rapid escape from the perceived threat (Losey \& Denno, 1998a; Humphreys \& Ruxton, 2019), but this behaviour can also carry shortand long-term fitness costs to the dropped individual such as: exposure to new predators (Losey \& Denno, 1998a), exposure to harsher environmental conditions (Ruth et al., 1975; Roitberg \& Myers, 1979), reduced feeding time (Roitberg et al., 1979; Johnson et al., 2007), increased development time (Agabiti et al., 2016), and reduced lifetime fecundity (Nelson et al., 2004; Nelson \& Rosenheim, 2006; Nelson, 2007; Agabiti et al., 2016). A display of tonic immobility immediately after dropping might serve to reduce localisation by predators but could also increase costs by lengthening exposure to harsher conditions and time without feeding (Miyatake et al., 2009; Humphreys \& Ruxton, 2018, 2019). Dropping has been studied most commonly in aphids (order Hemiptera), a group in which post-dropping tonic immobility is also exhibited (Bilska et al., 2018), and several abiotic and biotic factors have already been demonstrated to influence the trade-offs associated with the decision to drop (see Dill et al., 1990; Losey \& Denno, 1998a, b; and Braendle \& Weisser, 2001 for examples).

Characteristics of predators that influence the propensity to drop in various aphid species are: the relative size of a predator compared to its prey (Brown, 1974; Evans, 1976; Losey \& Denno, 1998a; Francke et al., 2008; Hoki et al., 2014), the predator species identity (Brown, 1974; Brodsky \& Barlow, 1986; Losey \& Denno, 1998a; Day et al., 2006; Hoki et al., 2014), and the predator's movement and/or foraging style (Brown, 1974; Brodsky \& Barlow, 1986; Losey \& Denno, 1998a; Day et al., 2006; Francke 
et al., 2008). For example, Francke et al. (2008) showed that older ladybirds (Harmonia axyridis), which were also larger and more active, induced more frequent dropping by pea aphids (Acyrthosiphon pisum) than younger ladybirds. Losey \& Denno (1998a) compared predator types and found that the coccinellid Coccinella septempunctata foraged more energetically than two smaller heteropteran predators, and initiated more than three-fold greater dropping by pea aphids. The underlying mechanisms for the links between predator size and activity and prey dropping behaviour may be the intensity of substrate vibration produced by the foraging predator, the encounter rate with aphids, the volume of aphid alarm pheromone released, or a combination of these (Montgomery \& Nault, 1977; Clegg \& Barlow, 1982; Francke et al., 2008). Additionally, predators sometimes become smeared with alarm pheromone (Mondor \& Roitberg, 2004), and movement of these predators will also be expected to increase aphid dropping (Francke et al., 2008).

Variability in the occurrence of dropping behaviour alone is not enough to fully evaluate a prey individual's antipredator response; the use of dropping might depend on an aphids' ability to use alternative defences. Aphids possess morphological and physiological defence adaptations, such as tubelike siphunculi on the abdomen which secrete waxy droplets and alarm pheromone. Droplets harden upon contact and can bind the appendages and mouthparts of predators and parasitoids (Dixon, 1958; Edwards, 1966; Outreman et al., 2005; Butler \& O'Neil, 2006; Pickett et al., 2007; Vandermoten et al., 2012). Some aphids also possess defensive structures such as frontal horns that can be used to attack predators (Arakaki, 1989; Pickett et al., 2007). Indirect defence also occurs for some aphids - myrmecophilous species form mutualisms with ants, which deter predators, and consequently exhibit reduced predator-avoidance behaviours (Depa et al., 2020). The deployment of behavioural defences can be more flexible, however, in response to the context of predation than morphological adaptations and indirect defences, and so the trade-offs associated with dropping will most valuably be weighed-up relative to alternative defensive behaviours. As well as dropping (Dixon, 1958; Roitberg \& Myers, 1978; Clegg \& Barlow, 1982; Agabiti et al., 2016; Harrison \& Preisser, 2016), aphids' behavioural responses to perceived predatory threat include: remaining motionless to avoid detection (Dixon, 1958, 1985; Brodsky \& Barlow, 1986), kicking (Dixon, 1958; Hartbauer, 2010; Dion et al., 2011; Polin et al., 2014), attacking with frontal horns (Arakaki, 1989), and walking away or backing-up 
(Dixon, 1958; Clegg \& Barlow, 1982; Brodsky \& Barlow, 1986). Some studies have already considered this broader portfolio of behaviours and found that when faced with larger, more mobile predators, dropping may be the most effective means of escape; but against the attacks of smaller, lessactive predators, aphids might be able to survive by exhibiting less costly behaviours such as kicking or walking away (Brown, 1974; Evans, 1976; Roitberg \& Myers, 1978). For example, Brodsky \& Barlow (1986) examined the defensive responses of pea aphids to syrphid larvae and coccinellid beetles. Syrphid larvae foraged more slowly than coccinellid beetles and, in response, aphids primarily backed-up. By contrast, coccinellid beetles foraged more vigorously, causing greater vibrations on the host plant, and most aphids that they encountered dropped off the plant, especially at higher temperatures when beetles were most active (Brodsky \& Barlow, 1986). Rather than individuals exhibiting a blanket defensive response against any threat, aphids can respond flexibly depending on the foraging rate, stage of development, method of attack, or species of a predator, and on the abiotic conditions.

Additional aspects of predator-prey interactions that could influence a prey individual's threat evaluation, and therefore propensity to utilise more extreme antipredator defences, include the proximity of the predator and whether the predator has made contact with the individual. Although many studies have explored the responses of aphids to attack, sometimes only behaviours that occur following physical contact with a natural enemy are recorded (Evans, 1976; Dion et al., 2011; Polin et al., 2014). Although contact can certainly be an important trigger for defensive behaviours, including dropping (Roitberg \& Myers, 1979; Brodsky \& Barlow, 1986; Dill et al., 1990; Losey \& Denno, 1998a; Dion et al., 2011), defence behaviours including dropping — can be initiated ahead of any contact (Clegg \& Barlow, 1982; Minoretti \& Weisser, 2000; Nelson \& Rosenheim, 2006; Harrison $\&$ Preisser, 2016). As touched on earlier, plant-borne vibrations and alarm pheromone detection can serve as alternative, or additional, triggers for dropping in aphids (Losey \& Denno, 1998a), and dropping in many taxa is known to take place at different stages of the predation sequence, as either a primary (pre-contact) or secondary (after physical contact with the predator) defence (Humphreys \& Ruxton, 2019). Yet studies do not tend to differentiate between pre- and post-contact dropping by aphids, although this could be important to understanding the threat level at which the decision to drop 
is taken. Similarly, where aphid dropping occurs in relation to a foraging predator is not often recorded, even though this would be expected to affect the sense of imminent threat experienced by the prey.

This current study explored a range of defensive behaviours exhibited by pea aphids and potato aphids (Macrosiphum euphorbiae) in response to two predator types with different foraging styles: two-spot ladybird (Adalia bipunctata) adults and lacewing (Chrysoperla carnea) larvae. Many studies record only the use of dropping as a defensive behaviour by aphids, but here we consider its use relative to alternative behaviours that may still be effective but less costly. Further, the defensive behaviour portfolios of aphids in response to different predatory threats have not been extensively explored for aphid species other than pea aphids, so here we also investigate potato aphids. Both the pea and potato aphid are in the Tribe Macrosiphini (Stekolshchikov \& Buga, 2020) but while the pea aphid specialises on legumes, the potato aphid is highly polyphagous and feeds on plants in many families (van Emden \& Harrington, 2007; AHDB, 2015). The two species also differ in the timing and stages of their life histories (AHDB, 2015), but both are commercially-important crop pests and virus vectors (van Emden \& Harrington, 2007). The differences in the biology and life history of the potato aphid make it a good model species to compare with its close relative: the pea aphid.

As part of exploring aphids' assessment of risk based on factors such as predator type, proximity and contact, and the use of dropping relative to other behavioural options, our study aimed to test two hypotheses:

1. Larger and faster-foraging predators generate greater vibrational movement, which can be an important component of aphids' threat detection. Different predator types also vary in their movements, mobility, and attack style. Therefore, we predict that the searching behaviour of ladybird adults will cause more frequent dropping by aphids than that of lacewing larvae, and that aphids will show differential use of various behavioural responses to the two predator types.

2. Dropping is the costliest defensive strategy for aphids, and as such it should only be used when threat is high-risk and imminent. Therefore, we predict that contact is an important trigger for dropping and that dropping will occur most commonly when a predator is in close proximity. 


\section{Materials and methods}

\subsection{Plant material}

Plants were grown from seed (Vicia faba cv The Sutton) or tubers (Solanum tuberosum cv Désirée) in a commercially produced insecticide-free compost mix (sand-perlite-peat mix containing N:P:K 17:10:15; William Sinclair Horticulture, Lincoln, UK) in a glasshouse with supplementary light (16:8 $\mathrm{h}$ light and $20: 15^{\circ} \mathrm{C}$ day:night) and watered daily. The aphid lines used in experiments were from established laboratory cultures held at the James Hutton Institute, Dundee: the pea aphid lines originated from wild lines collected in 2010 and the potato aphid lines originated from wild lines collected between 2014-2016.

\subsection{Insect rearing and maintenance}

Aphid clonal lines were reared on excised leaf material in ventilated containers comprising one Perspex cup (50 mm width $\times 150 \mathrm{~mm}$ depth) placed inside another; plant material was inserted through an approx. 5-mmdiameter circular hole in the base of the inner cup into approx. $10 \mathrm{~mm}$ depth of water in the base of the outermost cup, and the cup surface was sealed with a mesh-ventilated lid. Pea aphids (Acyrthosiphon pisum) from two different clonal lines were cultured on leaves from 2-3-week-old faba bean plants and potato aphids (Macrosiphum euphorbiae) from four different clonal lines were cultured on leaves from 3-week-old potato plants, with plant material refreshed weekly. Using this set up, age-synchronised cohorts of 3rd-4th instar nymphs were produced daily for experiments; these were 6-7 days old for A. pisum and 8-9 days old for M. euphorbiae.

Adult ladybirds and lacewing larvae were supplied by Dragonfli (Halstead, UK) and Ladybird Plantcare (Lewes, UK). Predators were maintained in large, ventilated Perspex cages $(90 \mathrm{~cm}$ height $\times 35 \mathrm{~cm}$ width $\times 45 \mathrm{~cm}$ depth) housed within a Snijders Scientific MicroClima growth chamber maintained on a light:dark cycle of $16: 8 \mathrm{~h}$ and $15: 10^{\circ} \mathrm{C}$, with a relative humidity of $60-70 \%$. Dry paper towels provided non-slippery substrate and shelter, and predators were supplied daily with $2-3$-week-old faba bean plants infested with an excess of pea aphids of a clonal line different to those used in experiments. Ladybirds were additionally supplied with dilute sugar water-soaked cotton wool. Two days prior to assays, predators were transferred (along with aphid-infested leaf material) to ventilated containers and 
placed in the glasshouse where experiments took place. Predators were then isolated into paper-towel lined ventilated cups and starved for $24-32 \mathrm{~h}$ ahead of assays.

\subsection{Experimental protocol}

Assays were conducted in a glasshouse with lighting conditions 16:8 h light:dark and heating conditions $20: 14^{\circ} \mathrm{C}$. The experimental set-up was positioned away from ventilation fans to minimise disturbance. For each assay, a plant pot (15 cm diameter) with two plant seedlings was encased by a mesh net supported by a metal frame and infested with 16 wingless immature aphids. Seedlings were trimmed pre-infestation if needed to prevent contact between seedlings and with the mesh net. Aphid-infested plants were left undisturbed overnight. Ten minutes before each assay, the pot was placed into a plastic tray $(6 \mathrm{~cm}$ height $\times 60 \mathrm{~cm}$ width $\times 39 \mathrm{~cm}$ depth $)$ and the frame and net removed. A JVC Everio HDD GZ-MG330AE hard disk camcorder fixed to an Ex-Pro ${ }^{\circledR}$ TR-654 professional photographic camera tripod (1350 mm height $\times 490 \mathrm{~mm}$ width) was angled to give a planar view of the pot.

Throughout trials, the experimenter wore a surgical mask to prevent plants or insects from being disturbed by air movement. All insects were transferred from cups to plants and vice versa using a fine artist's paintbrush. Each assay started at either $0900,1130,1400$, or $1630 \mathrm{~h}$. When filming began, the number of aphids remaining on plants was recorded using a Silverline 129472 telescopic inspection mirror to determine if any aphids had escaped overnight and/or fallen onto the soil. A predator (ladybird adult or lacewing larvae) was then added to the base of a randomly-selected seedling to start each assay.

Once each assay had started, the predator was observed for a 30-minute period, during which its movements and aphid behaviours deemed to be in response to the predator or its movements on the predator's nearby plant sections (considered as stem, petiole, petiolule, upper leaf or lower leaf) were dictated to the camera. Aphid behaviours were classified as: 'drop', 'walk' 'kick', 'shift', and 'no' response. 'Drop' was where an aphid dropped from its current plant section, usually to the substrate below. When drops occurred, it was also noted whether that aphid had been contacted by the predator and from what proximity to the predator the aphid dropped (immediately beside, same immediate plant section, same gross plant section, nowhere 
near but same seedling, or from the other seedling entirely). 'Walk' included behaviours ranging from backing-up a pace or two to running quickly away; drawing a distinct line between just backing-up and running to escape was difficult as often a back-up could transition to a walk and then a run. 'Kick' described the quick flicking of a leg or two legs in the direction of the predator. 'Shift' was defined as when aphids moved their body or antennae in response to the stimulus but without stepping anywhere; this category is not typically recorded in the literature, but it was deemed useful to differentiate between some slight motion/acknowledgement of predator presence and no response. 'No' was where the aphid remained motionless despite the predator having made contact.

If aphids departed from the pot by walking during the 30 minute observation period, they were not replaced. If an aphid dropped off the side of the pot (this happened mainly with the potato aphids on the larger potato plant seedlings), it was replaced on the substrate at the edge of the pot. If a predator climbed the rim of the pot, it was moved to the base of the seedling it had visited least recently (or not at all). If the predator did not encounter an aphid for five minutes it was also moved to the base of the least recent (or unexplored) seedling. If a predator successfully captured an aphid, it was given 10 minutes to finish consuming its prey and continue moving before it was replaced entirely with a predator of the same species. If a predator was motionless for two uninterrupted minutes or (ladybird-specific) flew away, it was also replaced with a fresh predator of the same species. After any occasion where a predator needed to be replaced, the next predator was introduced to the least recently-explored (or unexplored) seedling. The timing of the observation period was paused while predators were being replaced, and restarted when the new predator was on a seedling. Each predator was used only once. The observation period continued until 30 minutes had passed; the final predator, on some occasions still the initial predator, was then removed from the pot.

A total of 16 pea aphid assays took place in May 2019, eight with lacewing larvae as predators and eight with ladybird adults as predators, using pots of 2-4 week old bean cv. The Sutton plants. A total of 32 potato aphid assays took place in July 2019, 16 with lacewing larvae as predators and 16 with ladybird adults as predators, using pots of 2-3 week-old potato cv. Desirée plants. Behavioural Observation Research Interactive Software (BORIS) (Friard \& Gamba, 2016) was used to watch the video footage. 
Using the dictations of all predator movements and aphid responses on the predator's current plant section at any given moment in time, the antipredator behaviours in response to the predator's presence were coded. All statistical analyses were conducted using $\mathrm{R}$ version 4.0.3 (R Core Team, 2020). The analysis for Hypothesis 1 first involved a $2 \times 5$ extension of Fisher's exact test using the total counts of each response type exhibited with the two different predators. Standardised residuals were then calculated (Sharpe, 2015) for the responses to each predator type, using expected counts derived from the relative proportions of responses that the other predator type provoked. The predator types were then considered separately, using one-way analysis of means (ANOVA, not assuming equal variance) and post-hoc Games-Howell tests (suitable for unequal group sizes and variances) to compare the mean proportions of different responses per trial. The analysis for Hypothesis 2 involved three chi-square goodness-of-fit tests, comparing the frequencies of: (1) pre- and post-contact drops; (2) post-contact drops from different proximities to predators; and (3) pre-contact drops from different proximities to predators.

\section{Results}

\subsection{Hypothesis 1: ladybird adults cause more frequent dropping than} lacewing larvae, and aphids show differential use of behavioural responses to the two predator types

First, we considered the propensity of pea aphids to use different defences in response to the two predator types. A significant difference was found between the raw counts of aphid responses to ladybirds (drops: 61, walks: 69, kicks: 71 , shifts: 18 , and no response: 0 ) and to lacewings (drops: 4 , walks: 41 , kicks: 73 , shifts: 15 , and no response: 0$)(p<0.001)$. Comparison of the relative magnitudes of the standardised residuals indicated that lacewing larvae elicited significantly fewer drops (std. res. $=-5.43$ ) but significantly more kicks (std. res. $=4.55$ ) than would be predicted under the null hypothesis that pea aphids reacted to lacewing larvae in the same way as they reacted to ladybird adults. Correspondingly, ladybird adults were found to elicit significantly more drops (std. res $=21.20$ ) but significantly fewer kicks (std. res. $=-4.49$ ) than would be predicted under the null hypothesis that pea aphids reacted to them in the same way as they reacted to lacewing larvae. 
Focusing first on pea aphid responses to lacewing larvae, a significant difference was found in the mean proportion per trial of drops $(8.9 \%)$, walks (40.3\%), kicks (39.8\%), and shifts (10.9\%) exhibited by aphids (Figure 1a, $F=5.3971$, num $\mathrm{df}=3.000$, denom $\mathrm{df}=13.939, p<0.05)$; there were no cases of no response to contact. Between the proportions of these defences, aphids showed significantly more kicks compared with shifting $(p<0.05)$. Turning to the responses to ladybird adults, a significant difference was also found in the mean proportion per trial of drops (29.1\%), walks (34.9\%), kicks (25.6\%), and shifts (10.4\%) exhibited by aphids (Figure 1a, $F=7.0261$, num $\mathrm{df}=3.000$, denom $\mathrm{df}=15.003, p<0.01$ ); again there were no cases where pea aphids did not respond to contact with a predator. Between these defences, aphids showed significantly more dropping $(p<0.05)$ and walking $(p<0.01)$ than they did shifting behaviours.

Next, the propensity of potato aphids to use different defences in response to the two predator types was explored. As with the pea aphids, the proportion of raw counts of each defence was considered first. A significant difference $(p<0.05)$ was found between aphids' relative use of different responses to ladybirds (drops: 68, walks: 154, kicks: 81, shifts: 52, and no response: 4 ) and to lacewings (drops: 27 , walks: 72 , kicks: 65 , shifts: 27 , and no response: 5). In response to lacewing larvae, potato aphids were found to kick more than would be predicted (std. res. $=3.12$ ) based on expected values generated from the counts of behaviours in response to ladybird adults. In response to ladybird adults, potato aphids were found to drop more (std. res. $=2.64$ ) and kick less (std. res. $=-3.49$ ) than would be predicted based on expected values generated from the counts of behaviours in response to lacewing larvae.

Considering responses to lacewing larvae only, a significant difference was found in the mean proportion per trial of drops (14.3\%), walks (38.8\%), kicks (21.4\%), shifts (14.6\%) and lack of response to contact (11.0\%) exhibited by potato aphids (Figure $1 \mathrm{~b}, F=4.7725$, num $\mathrm{df}=4.000$, denom $\mathrm{df}=$ $36.451, p<0.01)$. Between these defences, potato aphids showed significantly more walking than dropping $(p<0.01)$, shifting $(p<0.01)$, and no response to contact $(p<0.05)$. Looking next at responses to ladybird adult predators, a significant difference was found in the mean proportion per trial of drops (20.9\%), walks (45.9\%), kicks (15.3\%), shifts (13.7\%) and lack of response to contact (4.2\%) exhibited by potato aphids (Figure 1b, $F=10.072$, num $\mathrm{df}=4.000$, denom $\mathrm{df}=36.275, p<0.001)$. Between 

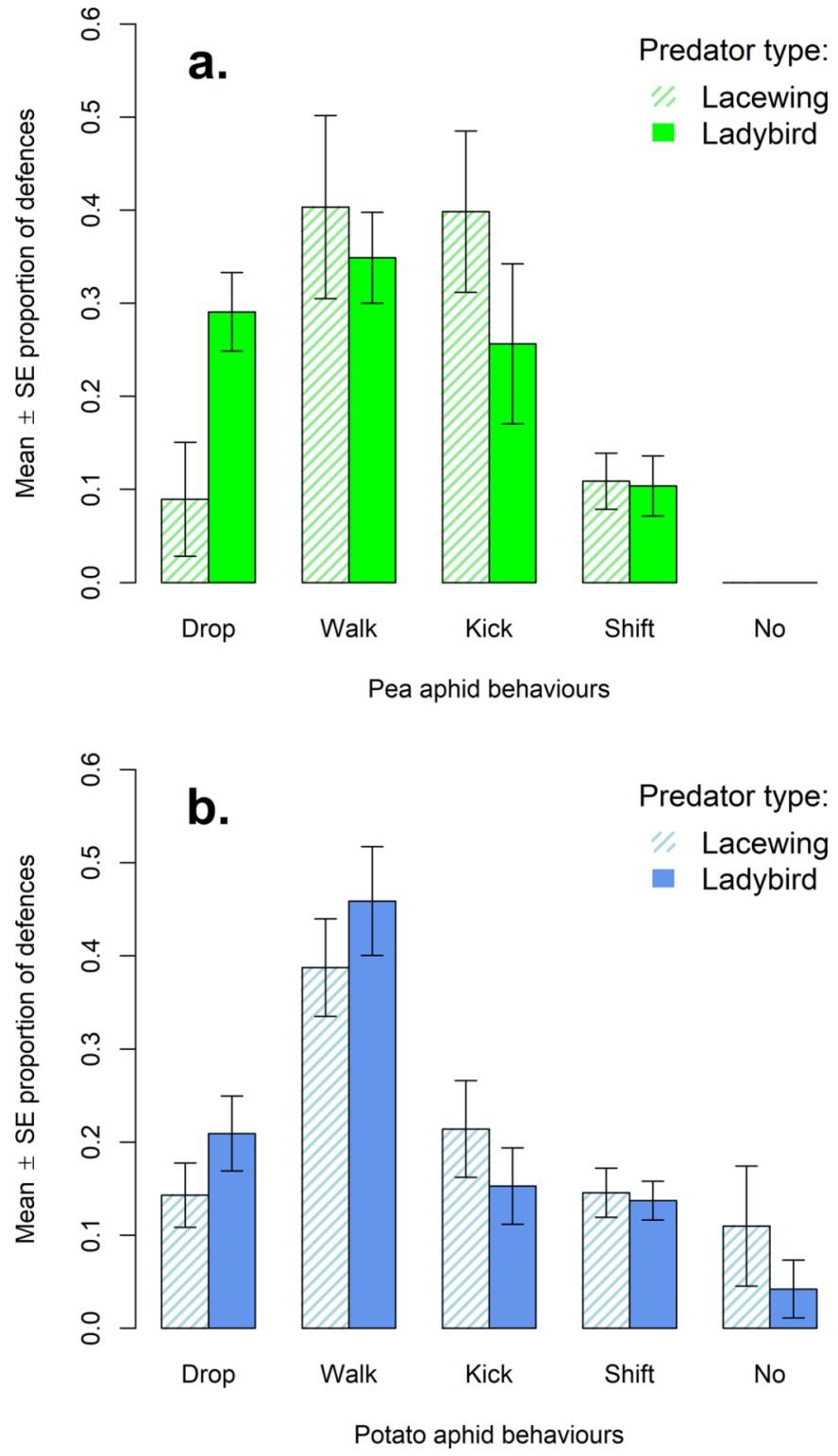

Figure 1. The mean \pm SE proportion of total defensive behaviours categorised as dropping, walking away/backing-up, kicking, shifting, and remaining motionless after contact exhibited by aphids in trials, where (a) shows the pea aphid trials with lacewing larvae ( $N=8$, dashed pale green bars) and with ladybird adults ( $N=8$, solid darker green bars), and (b) shows the potato aphid trials with lacewing larvae $(N=16$, dashed pale blue bars) and with ladybird adults $(N=16$, solid darker blue bars). 
these defences, potato aphids showed significantly more walking than they did dropping ( $p<0.05)$, kicking ( $p<0.01)$, shifting $(p<0.001)$, and no response to contact ( $p<0.001)$. Interestingly, though, in response to contact with foraging ladybirds, potato aphids also showed significantly more dropping than no response $(p<0.05)$. In response to both predators, then, walking away was the most prevalent defence of potato aphids in terms of raw occurrence. But in response to ladybirds, aphids dropped significantly more than they showed no response to contact (the cases of no response to contact translated into those aphids being grabbed by predators).

\subsection{Hypothesis 2: contact is an important trigger for dropping, and} dropping will occur most commonly when a predator is in close proximity

Considering the dropping behaviour deemed to result from nearby predator presence, pea aphids dropped significantly more commonly post-contact $(N=44)$ than pre-contact $(N=5)\left(\chi_{1}^{2}=31.041, p<0.001\right)$. Of the cases where pea aphids dropped post-contact with a predator, 39 occurred from adjacent to the predator, three from the same plant section as the predator, and two from the same gross plant section as the predator. A significant difference was found between the frequencies of post-contact drops from these three recorded proximities $\left(\chi_{2}^{2}=60.591, p<0.001\right)$, with the vast majority of drops starting from immediately beside the predator. There were only five cases of pea aphids dropping pre-contact with a predator: four of these aphids dropped from the same plant section as the predator and one dropped from the same gross plant section. The small sample size meant that a significant difference could not be detected between the frequency of pre-contact drops from these two proximities $\left(\chi_{1}^{2}=1.8, p=0.1797\right)$, but it seemed that when aphids dropped pre-contact it was primarily from the same plant section as the predator.

Turning to the potato aphids, considering the dropping behaviour deemed to result from nearby predator presence, potato aphids also dropped significantly more commonly post-contact $(N=77)$ than pre-contact $(N=11)$ $\left(\chi_{1}^{2}=49.5, p<0.001\right)$. Of the cases where potato aphids dropped postcontact with a foraging predator: seventy drops occurred from immediately beside the predator, five from the same plant section as the predator, and two from the same gross plant section as the predator. A significant difference was found between the frequencies of post-contact drops from these three recorded proximities $\left(\chi_{2}^{2}=115.04, p<0.001\right)$, with the vast majority of 
drops starting from immediately beside the predator. There were only eleven cases of potato aphids dropping pre-contact with a predator, but where the dropping was still interpreted as being in response to the presence of the predator as opposed to some other unknown trigger: eight of these aphids dropped from the same plant section as the predator, two dropped from the same gross plant section, and one dropped from right beside the predator. A significant difference was found between the frequency of pre-contact drops from these three proximities $\left(\chi_{2}^{2}=7.8182, p<0.05\right)$. Even though the small sample sizes meant that this test might be unreliable, it illustrates a tendency shown by potato aphids that pre-contact drops occurred primarily from the same plant section as the predator, as was shown for pea aphids.

\section{Discussion}

Overall, the study collected data on the range of behaviours both pea and potato aphids exhibit in response to two major predatory threats, finding that dropping was an important component within both aphids' defensive behavioural repertoires. Considering first Hypothesis 1, both species dropped significantly more commonly (relative to other defences) when faced with ladybird adult predators than lacewing larvae predators - lacewing larvae correspondingly elicited a significantly greater proportion of kicking defence. In pea aphids, dropping made up a similar proportion of the total behavioural responses as walking away and kicking in response to ladybird adults, but dropping occurred significantly less frequently when aphids were faced with lacewing larvae: drops constituted around a three times smaller proportion of the total defensive behaviours exhibited and occurred at a fifteen times smaller raw frequency. Pea aphids rarely shifted when faced with either predator, and never remained motionless after any predator contact. In potato aphids, dropping made up a similar proportion of the total behavioural responses as kicking, but a significantly smaller proportion than walking away in response to ladybird adults. Dropping made up a similar proportion of responses as shifting and remaining motionless after contact in response to lacewing larvae, where again walking away was by far the most common response. Although walking away was the primary response of potato aphids to both predator types, dropping still constituted an important component of their defensive response and differentiation in its usage was still seen between the two predator types. 
Predator species (Brown, 1974; Harmon et al., 1998; Hoki et al., 2014) and predator functional types (Brodsky \& Barlow, 1986; Losey \& Denno, 1998a; Day et al., 2006) vary in the way that they search plants and thereby elicit different responses in their prey. Pea aphids have previously been found to drop more frequently from host plants in response to coccinellid beetles compared to syrphid larvae, an effect that was attributed to the energetic and vibrational differences in predator foraging styles (Brodsky \& Barlow, 1986). In this present study, lacewing larvae predators appeared to match more closely the syrphid larvae style of foraging, in that they were generally slow-moving, while ladybird adults fitted the typical behaviour of coccinellids being fast-paced predators. This might explain why dropping made up a greater proportion of both aphid species' responses to ladybird adults these predators were more energetic, perhaps causing more vibrations across plants, and more likely to attack quickly, therefore the most extreme defence of dropping might be more necessary to evade capture. By contrast, with slower-foraging lacewing larvae there might have been fewer plant vibrations, attacks were perhaps less sudden, and other defences such as walking away or kicking might be effective without aphids resorting to dropping. Predator type, or at least foraging style, seemed an important factor that influenced dropping propensity, and likely feeds into aphids' assessment of predation risk. Additional experiments would be needed, using multiple predator types with comparable body sizes and foraging speeds, to confirm that the differing reactions of aphids to these two predator types were a consequence of these predator characteristics rather than morphological or chemical signalling traits. Ladybird adults also encountered more aphids than lacewing larvae, in part because of their quicker pace around plants allowing for more exploration, which may account for the significantly higher raw number of drops and total defences they provoked in both aphid species.

Turning to the first part of Hypothesis 2, considering contact with a predator as another factor that aphids may use to assess risk, both aphid species were found to drop significantly more following contact with a predator than before contact. Further, most post-contact drops were initiated from adjacent to the predator, often immediately following contact. Clearly contact by a predator appears to act as a key trigger for dropping, the most extreme escape defence in terms of potential costs, as has been previously reported (Roitberg et al., 1979; Dill et al., 1990; Nelson \& Rosenheim, 2006; Polin et 
al., 2014). It is unsurprising that contact with a predator would trigger immediate dropping - and therefore from close proximity - as it is an indicator of imminent predation threat. It is, however, interesting that aphids delay their dropping escape until such contact is made. We think this is unlikely to be due to aphids being unable to sense the presence of predators ahead of contact, as they would likely detect their movement through plant-borne vibrations as well as visually. Rather, we propose that aphids only drop postcontact because their predators often do not pose a direct danger and because the costs of dropping (compared to kicking or walking away) are high. In this study, predators rarely headed clearly towards a specific target aphid, rather they seemed to roam about plant sections without noticing that aphids were present until they incidentally contacted them. Most aphids, particularly pea aphids, also survived the first instances of contact with a predator and were able to react before being subdued, indicating that predators rarely made quick and decisive attacks on prey. It may, therefore, be adaptive for aphids to wait until contact has been made before dropping to escape, as: (1) there is a high chance that the predator will not notice them at all, so they need not interrupt their feeding; (2) the first contact from a predator does not result in complete subjugation and so the aphid would likely have time to respond before the predator attacks; and (3) aphids benefit from avoiding the potentially substantial costs associated with dropping (Roitberg \& Myers, 1979; Dill et al., 1990; Losey \& Denno, 1998a; Nelson et al., 2004; Nelson \& Rosenheim, 2006; Nelson, 2007; Agabiti et al., 2016).

Exploring the predator proximity aspect of Hypothesis 2 further, occasionally aphids did drop pre-contact, but these drops mostly still took place from the same plant section that the predator was exploring. It might make sense that aphids wait until a predator moves onto the same section and poses a direct threat so that potentially costly pre-contact dropping is warranted, but if making a pre-emptive drop then they should not wait until the predator is immediately beside them in case they are subdued. Alternatively, or additionally, regardless of whether or not a predator is in close proximity, if an aphid senses a change in vibration frequency that is sufficiently large to indicate risk, perhaps it will drop even if other triggers (i.e., predator contact) have not been elicited. Throughout this study, there were also a handful of drops by aphids that were not included in the analysis because they were not deemed to have been directly due to the movement or behaviour of the predator. Some of these for both pea and potato aphids were likely triggered 
by alarm pheromone detection as the drops coincided with periods when an aphid was being consumed or had a limb removed by a predator elsewhere on the plant. If alarm pheromone warns of predatory attack, dropping from a relatively long-distance may be adaptive where it enables aphids to escape a plant or area which has at least one predator present. The fact that pheromone is released may be enough to raise the perceived threat levels such that the predator need not make contact or even be present on the aphid's same plant section to trigger dropping. Other drops were noticed where pea aphids had recently dropped but then seemed to drop again as they were climbing back onto plants, but without any obvious further threat from the predator. If, as assumed, these drops were triggered more by aphids' recent experience of threat and continuing stressed state rather than a new escape-inducing stimulus, it is unsurprising that many of these did not occur anywhere near the predator. Further excluded drops by both pea and potato aphids occurred for unknown reasons at relatively long distances from predators; perhaps these drops did not serve an antipredator function, rather they might have been triggered by a thermoregulatory need, or a desire to locate a different food source. Future studies with a greater ability to track all individuals and monitor microclimates and alarm pheromone presence might shed further light on the various triggers and functions of dropping.

Finally, by examining the defensive behaviour of the potato aphid as well as that of the pea aphid, our findings indicate that there are important intraspecific differences. Although developmental stage, a factor which has been seen to influence the propensity to drop in aphids (Losey \& Denno, 1998a; Gish \& Inbar, 2006; Gish et al., 2012), was not controlled for across the species, the differences in relative use of different behaviours might reflect differences in their biology or life history. Pea aphids appeared to be more sensitive to the processes that trigger dropping, be they vibrations, visual cues, or contact with a predator. It is plausible that due to their different sizes, morphologies, detectability on host plants, and/or relative encounter rates with different natural enemies, pea and potato aphids have evolved different sensitivities to triggers of dropping that are adaptive to their own typical circumstances. For example, if these two species vary in their typical encounter rate with visual predators, this would influence which behaviours are most likely to be effective during predator-prey encounters. Some adult coccinellids do use vision as an important guide to their foraging (Nakamuta, 1984), but different species vary in their dependence on visual cues 
(Harmon et al., 1998). If pea aphids are more likely to be detected visually by their most common natural enemies than potato aphids, and their predators are more likely to be successful in locating them on plants, then dropping might be a more essential component of their behavioural repertoire. However, further study into the relative sensitivity of aphids to triggers of dropping and their predators' prey detection abilities under varying abiotic conditions is needed to understand interspecific behavioural differences more fully. Additional experiments utilising aphid lines freshly sourced from the wild would also be valuable in confirming how closely the antipredator behaviours observed in this study reflect those shown by aphids of either species when they are not reared in the absence of natural predators.

In conclusion, dropping appears to be a defence deployed only when the risk of imminent predation is assessed as being high, where factors that might increase the propensity of aphids to drop include: faster-foraging predators such as adult ladybirds, predator proximity, and contact with a predator that may trigger more targeted, intensive search by the predator. Dropping can carry fitness costs (Roitberg et al., 1979; Dill et al., 1990; Nelson, 2007; Agabiti et al., 2016), and so flexible use of it as an extreme defence only when the predation risk is assessed as being high would be adaptive. Future research building on what is already known about post-dropping tonic immobility (Niku, 1975; Wohlers, 1981; Bilska et al., 2018; Humphreys \& Ruxton, 2018) and aphid return onto plants post-dropping (Niku, 1975, 1976; Roitberg et al., 1979; Gish \& Inbar, 2006) will help to develop an even fuller picture of the trade-offs involved with dropping. Additionally, factors relating to the predatory threat that might influence dropping likelihood also warrant more detailed investigation, for example predator approach trajectory. Several aphid species have been seen to walk away from predators (instead of dropping) more frequently when approached from the front rather than from the rear (Dixon, 1958; Hajek \& Dahlsten, 1987). Other aphids may also be less likely to drop from host plants if they have a clear visual warning of an approaching predator and time to walk away pre-contact. Studying the impact of multiple predator types in a single predation situation would also prove valuable. More broadly, future research into dropping behaviour or indeed any other antipredator defence - in any prey species may benefit from investigating the use of a specific defence within the context of the available antipredator portfolio. Understanding the decision-making that might drive the use of one defence over alternatives is key to shedding light 
on the various costs and benefits of all defences and the selective pressures that could have led to their development over evolutionary time.

\section{Acknowledgements}

We thank two anonymous referees for their helpful and insightful comments. RKH was funded by the Perry Foundation and the University of St Andrews. AJK is supported by the strategic research programme funded by the Scottish Government's Rural and Environment Science and Analytical Services Division. We thank several members of the James Hutton Institute (Dundee), without whom this work would not have been possible: Carolyn Mitchell for invaluable advice on and assistance with insect rearing, Jim Wilde for the supply and maintenance of plant material, and Dave Gray for technical assistance with the Snijders Scientific MicroClima growth chamber. Thanks also to Gaynor Malloch (James Hutton Institute, Dundee), Daniel Leybourne, Desiré Macheda, and Pilar Morera Margarit for advice on and assistance with aphid rearing, and to Elvyra Bulavaite for advice on maintenance of ladybirds and lacewings. Further thanks go to Julia Ferrari (University of York, UK) for supplying the pea aphid lines and the James Hutton Institute (Dundee) for supplying the potato aphid lines used in experiments.

\section{References}

Agabiti, B., Wassenaar, R.J. \& Winder, L. (2016). Dropping behaviour of pea aphid nymphs increases their development time and reduces their reproductive capacity as adults. PeerJ 4: e2236.

AHDB (2015). Encyclopaedia of pests and natural enemies in field crops. - Agriculture and Horticulture Development Board, Coventry, p. 123.

Arakaki, N. (1989). Alarm pheromone eliciting attack and escape responses in the sugar cane woolly aphid, Ceratovacuna lanigera (Homoptera, Pemphigidae). — J. Ethol. 7: 83-90.

Bilska, A., Francikowski, J., Wyglenda, A., Masłowski, A., Kaszyca, N. \& Depa, Ł. (2018). Aphids playing possum - defensive or mutualistic response? - J. Insect Behav. 31: 42-53.

Braendle, C. \& Weisser, W.W. (2001). Variation in escape behavior of red and green clones of the pea aphid. - J. Insect Behav. 14: 497-509.

Brodsky, L.M. \& Barlow, C.A. (1986). Escape responses of the pea aphid, Acyrthosiphon pisum (Harris) (Homoptera, Aphididae) - influence of predator type and temperature. Can. J. Zool. 64: 937-939.

Brown, H.D. (1974). Defensive behavior of the wheat aphid Schizaphis graminum Hemiptera Homoptera Aphididae against Coccinellidae. — J. Entomol. Ser. A-Gen. 48: 157-165. 
Butler, C.D. \& O'Neil, R.J. (2006). Defensive response of soybean aphid (Hemiptera: Aphididae) to predation by insidious flower bug (Hemiptera: Anthocoridae). - Ann. Entomol. Soc. Am. 99: 317-320.

Caro, T. (2005). Antipredator defenses in birds and mammals. - University of Chicago Press, Chicago, IL.

Clegg, J.M. \& Barlow, C.A. (1982). Escape behavior of the pea aphid Acyrthosiphon pisum (Harris) in response to alarm pheromone and vibration. - Can. J. Zool. 60: 2245-2252.

Day, K.R., Docherty, M., Leather, S.R. \& Kidd, N.A.C. (2006). The role of generalist insect predators and pathogens in suppressing green spruce aphid populations through direct mortality and mediation of aphid dropping behavior. — Biol. Control 38: 233-246.

Depa, Ł., Kaszyca-Taszakowska, N., Taszakowski, A. \& Kanturski, M. (2020). Ant-induced evolutionary patterns in aphids. — Biol. Rev. 95: 1574-1589.

Dill, L.M., Fraser, A.H.G. \& Roitberg, B.D. (1990). The economics of escape behavior in the pea aphid, Acrythosiphon pisum. - Oecologia 83: 473-478.

Dion, E., Polin, S.E., Simon, J.C. \& Outreman, Y. (2011). Symbiont infection affects aphid defensive behaviours. — Biol. Lett. 7: 743-746.

Dixon, A.F.G. (1958). The escape responses shown by certain aphids to the presence of the coccinellid Adalia decempunctata (L.). — Trans. Roy. Entomol. Soc. Lond. 110: 319-334.

Dixon, A.F.G. (1985). Aphid ecology. — Blackie and Son, Glasgow.

Edmunds, M. (1974). Defence in animals: a survey of anti-predator defences. - Longman, Burnt Mill.

Edwards, J.S. (1966). Defence by smear: supercooling in the cornicle wax of aphids. Nature 211: 73-74.

Evans, H.F. (1976). Role of predator-prey size ratio in determining efficiency of capture by Anthocoris nemorum and escape reactions of its prey, Acyrthosiphon pisum. - Ecol. Entomol. 1: 85-90.

Francke, D.L., Harmon, J.P., Harvey, C.T. \& Ives, A.R. (2008). Pea aphid dropping behavior diminishes foraging efficiency of a predatory ladybeetle. - Entomol. Exp. Appl. 127: 118-124.

Friard, O. \& Gamba, M. (2016). BORIS: a free, versatile open source event logging software for video/audio coding and live observations. - Methods Ecol. Evol. 7: 1325-1330.

Gish, M., Dafni, A. \& Inbar, M. (2012). Young aphids avoid erroneous dropping when evading mammalian herbivores by combining input from two sensory modalities. - PLoS ONE 7: e32706.

Gish, M. \& Inbar, M. (2006). Host location by apterous aphids after escape dropping from the plant. — J. Insect Behav. 19: 143-153.

Hajek, A.E. \& Dahlsten, D.L. (1987). Behavioral interactions between three birch aphid species and Adalia bipunctata larvae. - Entomol. Exp. Appl. 45: 81-87.

Harmon, J.P., Losey, J.E. \& Ives, A.R. (1998). The role of vision and color in the close proximity foraging behavior of four coccinellid species. - Oecologia 115: 287-292.

Harrison, K.V. \& Preisser, E.L. (2016). Dropping behavior in the pea aphid (Hemiptera: Aphididae): how does environmental context affect antipredator responses? - J. Insect Sci. 16: 89. 
Hartbauer, M. (2010). Collective defense of Aphis nerii and Uroleucon hypochoeridis (Homoptera, Aphididae) against natural enemies. — PLoS ONE 5: 13.

Hoki, E., Losey, J. \& Ugine, T.A. (2014). Comparing the consumptive and non-consumptive effects of a native and introduced lady beetle on pea aphids (Acyrthosiphon pisum). Biol. Control 70: 78-84.

Humphreys, R.K. \& Ruxton, G.D. (2018). A review of thanatosis (death feigning) as an antipredator behaviour. - Behav. Ecol. Sociobiol. 72: 22.

Humphreys, R.K. \& Ruxton, G.D. (2019). Dropping to escape: a review of an underappreciated antipredator defence. - Biol. Rev. 94: 575-589.

Johnson, M.L., Armitage, S., Scholz, B.C.G., Merritt, D.J., Cribb, B.W. \& Zalucki, M.P. (2007). Predator presence moves Helicoverpa armigera larvae to distraction. - J. Insect. Behav. 20: 1-18.

Lima, S.L. \& Dill, L.M. (1990). Behavioral decisions made under the risk of predation: a review and prospectus. — Can. J. Zool. 68: 619-640.

Losey, J.E. \& Denno, R.F. (1998a). The escape response of pea aphids to foliar-foraging predators: factors affecting dropping behaviour. — Ecol. Entomol. 23: 53-61.

Losey, J.E. \& Denno, R.F. (1998b). Interspecific variation in the escape responses of aphids: effect on risk of predation from foliar-foraging and ground-foraging predators. - Oecologia 115: 245-252.

Minoretti, N. \& Weisser, W.W. (2000). The impact of individual ladybirds (Coccinella septempunctata, Coleoptera: Coccinellidae) on aphid colonies. - Eur. J. Entomol. 97: 475-479.

Miyatake, T., Nakayama, S., Nishi, Y. \& Nakajima, S. (2009). Tonically immobilized selfish prey can survive by sacrificing others. - Proc. Roy. Soc. Lond. B: Biol. Sci. 276: 27632767.

Mondor, E.B. \& Roitberg, B.D. (2004). Inclusive fitness benefits of scent-marking predators. - Proc. Roy. Soc. Lond. B: Biol. Sci. 271: S341-S343.

Montgomery, M.E. \& Nault, L.R. (1977). Comparative response of aphids to alarm pheromone, (E)-beta-farnesene. — Entomol. Exp. Appl. 22: 236-242.

Nakamuta, K. (1984). Visual orientation of a ladybeetle, Coccinella septempunctata L., (Coleoptera: Coccinellidae), toward its prey. - Appl. Entomol. Zool. 19: 82-86.

Nelson, E.H. (2007). Predator avoidance behavior in the pea aphid: costs, frequency, and population consequences. - Oecologia 151: 22-32.

Nelson, E.H., Matthews, C.E. \& Rosenheim, J.A. (2004). Predators reduce prey population growth by inducing changes in prey behavior. - Ecology 85: 1853-1858.

Nelson, E.H. \& Rosenheim, J.A. (2006). Encounters between aphids and their predators: the relative frequencies of disturbance and consumption. - Entomol. Exp. Appl. 118: 211219.

Niku, B. (1975). Verhalten und fruchtbarkeit ungeflügelter erbsenläuse (Acyrthosiphon pisum) nach einer fallreaktion. — Entomol. Exp. Appl. 18: 17-30.

Niku, B. (1976). Some consequences of the drop reaction of Acyrthosiphon pisum for the larvae of Syrphus corollae. - Entomophaga 21: 257-264. 
Outreman, Y., Le Ralec, A., Wajnberg, E. \& Pierre, J.-S. (2005). Effects of within- and among-patch experiences on the patch-leaving decision rules in an insect parasitoid. Behav. Ecol. Sociobiol. 58: 208-217.

Pickett, J.A., Bruce, T.J.A. \& Glinwood, R.T. (2007). Chemical ecology. — In: Aphids as crop pests (van Emden, H.F. \& Harrington, R., eds). CABI, Trowbridge, p. 148-172.

Polin, S., Simon, J.-C. \& Outreman, Y. (2014). An ecological cost associated with protective symbionts of aphids. - Ecol. Evol. 4: 836-840.

R Core Team (2020). R: a language and environment for statistical computing. - R Foundation for Statistical Computing, Vienna.

Roitberg, B.D. \& Myers, J.H. (1978). Adaptation of alarm pheromone responses of pea aphid Acyrthosiphon pisum (Harris). — Can. J. Zool. 56: 103-108.

Roitberg, B.D. \& Myers, J.H. (1979). Behavioural and physiological adaptations of pea aphids (Homoptera: Aphididae) to high ground temperatures and predator disturbance. Can. Entomol. 111: 515-519.

Roitberg, B.D., Myers, J.H. \& Frazer, B.D. (1979). The influence of predators on the movement of apterous pea aphids between plants. - J. Anim. Ecol. 48: 111-122.

Ruth, W.E., McNew, R.W., Caves, D.W. \& Elkenbary, R.D. (1975). Greenbugs (Hom.: Aphididae) forced from host plants by Lysiphlehus testaceipes (Hym.: Braconidae). — Entomophaga 20: 65-71.

Ruxton, G.D., Allen, W.L., Sherratt, T.N. \& Speed, M.P. (2018). Avoiding attack: the evolutionary ecology of crypsis, warning signals and mimicry. - Oxford University Press, Oxford.

Sharpe, D. (2015). Your chi-square test is statistically significant: now what? — Pract. Assess. Res. Evaluation 20: 1-10.

Stekolshchikov, A. \& Buga, S. (2020). An analysis of existing views on the taxonomic system of the tribe Macrosiphini. — Ann. Up. Siles. Mus. (Ent.) 29: 1-18.

Van Emden, H.F. \& Harrington, R. (2007). Aphids as crop pests. - CABI, Trowbridge.

Vandermoten, S., Mescher, M.C., Francis, F., Haubruge, E. \& Verheggen, F.J. (2012). Aphid alarm pheromone: an overview of current knowledge on biosynthesis and functions. Insect Biochem. Mol. 42: 155-163.

Wohlers, P. (1981). Effects of the alarm pheromone (E)- $\beta$-farnesense on dispersal behaviour of the pea aphid Acyrthosiphon pisum. — Entomol. Exp. Appl. 29: 117-124. 